\title{
Sex Differences in Enzyme Activities Relating to Cholesteryl Ester Metabolism in Aorta and Liver from SHRSP, SHR, and WKR during aging
}

\author{
Takako Tomita, Yasuhumi ShIrasaki, Ikumi Yonekura \\ and Eiichi HaYashi
}

It has been commonly assumed that sex hormons have great influences on lipid metabolism which is closely related to atherosclerosis. In the course of studying the mechanism of sex difference in atherogenesis, the differences in various enzyme activities, especially relating to cholesteryl ester metabolism was investigated in SHRSP, SHR, and WKR during aging.

SHRSP, SHR, and WKR originally from Dr. Okamoto of Kinki University were maintained for 1,6 , and 11 months after weaning on CMF diet from Oriental Yeast $\mathrm{Co}$, and killed between 9-12 AM after 12h of fasting. The enzymes measured are aortic cholesterylester hydrolase and triacylglycerol hydolase, liver cholesterylester hydrolase and acid phosphatase in lysosomal fraction and cholesterylester synthetase in microsomal fraction.

Aortic acidic cholesterylester hydrolase was measured in the supernatant of the aorta homogenate, while liver cholesterylester hydrolase was measured in the lysosomal fraction homogenized by Polytron. The assay method was reported at the last meeting. For the assay of liver cholesterylester synthetase activity, microsomal fraction was incubated for $2 \mathrm{~h}$ with $\left[{ }^{14} \mathrm{C}\right]$-oleate, $\mathrm{CoA}, \mathrm{ATP}$, and $\mathrm{MgCl}_{\mathbf{2}}$ in $0.01 \mathrm{M}$ Tris buffer ( $\mathrm{pH} 7.4$ ) and incorporation of the radioactivity into cholesterylester fraction was measured. The produced cholesterylester was separated from the substrate by a silicic acid column using $15 \%$ hexane in benzene as the eluant.

In order to examine how and at what age sex difference began to appear, the enzyme activities were compared between male and female rats of Wistar Strain at age of $1,2.5$, and 6 months after birth. Although there was no sex difference in the activities at month 1 , aortic and liver cholesterylester hydrolase and liver cholesterylester synthetase activities were significantly higher in female rats at month 2.5 and 6 . On the other hand, liver acid phosphatase activity tended to be higher in male rats through the whole period. Therefore, measurement of the enzyme activities in SHRSP, SHR, and WKR were made at 1,6 and 11 months after weaning.

Aortic cholesterylester hydrolase activity in female SHRSP was much higher than that in the male rats at month 1 after weaning, whereas the sex difference completely diminished at month 6 and 11 as the activity gradually decreased with aging. The activity in the male SHR and WKR was also significantly higher at month 1 and the sex difference was still observed at month 6 and 11 contrasting to SHRSP. Just like aortic cholesterylester hydrolase, aortic triacylglycerol hydrolase

From the Department of Pharmacology, Shizuoka College of Pharmaceutical Sciences, Shizuoka. 
activity in SHRSP was much greater than that in male rats at month 1 after weaning but the sex difference was not observed at month 6 and 11. The activity in female SHR and WKR was always significantly higher.

Sex difference in liver cholesterylester hydrolase activity of SHRSP differs from that of aortic cholesterylester hydrolase in the followings ; 1) sex difference was much greater in liver than in aorta, 2) sex difference was observed through the whole period, and 3 ) the decrease in the activity due to aging was not so sharp. Sex difference in SHR and WKR was also more remarkable in liver than in aorta.

Microsomal cholesterylester synthetase in liver was also influenced by sex. Female rats had the greater activity than male rats in all strains through the whole period. In case of SHR and WKR, the sex difference became more evident due to aging. Contrasting to cholesterylester hydrolase, cholesterylester synthetase activity increased gradually due to aging in SHRSP and SHR, especially the increase in SHRSP at month 11 was marked. Liver acid phosphatase, a marker enzyme of lysosome was influenced oppositely by sex. Generally speaking, male rats had the higher activity than female rats, and the activity was almost unchanged through the period.

In order to see whether the sex difference observed was mediated by sex hormons, the change due to castration of the enzyme activities was studied. Male and female rats of Wistar Strain at age of 2 months were submitted to castration, and 2 weeks after, the enzyme activities were compared between castrated and sham operated rats. Due to castration, aortic cholesterylester hydrolase activity sharply decreased in female rats, while the activity increased in male rats. Liver cholesterylester hydrolase activity significantly increased in castrated male rats. However liver acid phosphatase was not altered in both male and female rats due to castration. Intramuscular injection of $17 \beta$-estradiol $(1 \mathrm{mg} / \mathrm{Kg} / \mathrm{day})$ to 2.5 months old rats for 2 weeks significantly increased liver cholesterylester synthetase and cholesterylester hydrolase activities, whereas it had no effect on liver acid phosphatase.

The results suggest that the sex difference observed, specifically on the enzyme activities relating to cholesteryl ester metabolism is mediated by sex hormons, and sex hormons may play an important role on aortic lipid metabolism by regulating these enzyme activities. 